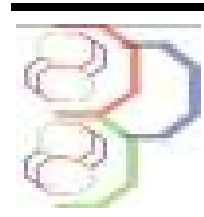

Journal of Applied Biosciences 67:5289 - 5301

\title{
Étude et prise en compte en modélisation de l'effet de la latitude sur la réponse à la photopériode chez divers génotypes de mil (Pennisetum glaucum (L.) R. Br.) du Sénégal
}

\author{
Kouakou ${ }^{1,2,4}$ PK, Muller ${ }^{1,3,6}$ B, Guissé 2 A, Yao ${ }^{4}$ RN, Fofana 5 A, Cissé ${ }^{1}$ N \\ ${ }^{1}$ CERAAS-ISRA, BP 3320 Thiès Escale, Sénégal \\ 2 UCAD, Avenue Cheikh Anta Diop, BP 5005 Dakar, Sénégal \\ ${ }^{3}$ CIRAD, Umr Agap, F-34398 Montpellier, France \\ 4 INP-HB, BP 1093 Yamoussoukro, Côte d'Ivoire \\ ${ }^{5}$ CNRA-ISRA, BP 53 Bambey, Sénégal \\ ${ }^{6}$ AfricaRice, Station Régionale du Sahel, BP 96 Saint-Louis, Sénégal \\ Adresse du principal auteur : patrice.kouakou@inphb.edu.ci
}

Original submitted in on $16^{\text {th }}$ April 2013 Published online at www.m.elewa.org on 30 th July 2013. https://dx.doi.org/10.4314/iab.v67i0.95051

\section{RESUME}

Objectif : L'étude a porté sur la caractérisation des variations de cycles de différents génotypes de mil cultivés au Sénégal sous différentes latitudes et l'amélioration de la modélisation de leur réponse à la photopériode. Méthodologie et résultat : Des essais à plusieurs dates de semis (12 juin, 10 juillet et 08 août) ont été conduits en 2012 sur 3 sites (Ndiol, Bambey et Kolda) au Sénégal dans un dispositif en blocs complets randomisés à 3 répétitions, et sous de bonnes conditions d'alimentation hydrique et de fertilité. Le matériel végétal était composé de 2 variétés améliorées, de type Souna, et de 6 écotypes locaux dont 2 de type Souna et 4 de type Sanio, qui ont été collectés suivant un gradient latitudinal nord-sud en mai 2011. Une caractérisation des durées semis-feuille drapeau de ces génotypes a été faite en fonction des dates de semis et de la latitude. Le modèle Impatience a été paramétrée puis utilisé pour simuler les dates d'initiation paniculaire sur les sites. Les résultats ont montré que les durées semis-feuille drapeau diminuaient avec les semis tardifs et augmentaient avec la latitude, avec une différence entre sites plus marquée pour les semis précoces. Le paramétrage du modèle Impatience obtenu à Bambey ne permettait pas de simuler correctement les durées de la phase végétative à Ndiol et à Kolda. Une équation de correction a été proposée pour estimer les paramètres du modèle à Ndiol et à Kolda en prenant en compte les latitudes de ces sites et le paramétrage du modèle à Bambey. Cette procédure a permis de simuler correctement les dates d'initiation paniculaire de ces sites avec des valeurs de RMSE proches de celles obtenues si le paramétrage spécifique de chacun de ces sites était utilisé.

Conclusion et application : Cette étude a permis de montrer que la plupart des variétés traditionnelles, précoces comme tardives, sont plus ou moins photosensibles. La méthode de correction du Psens proposée a permis d'améliorer les performances du modèle. Ce résultat trouve une application opérationnelle en modélisation des cultures et ses applications dont la prévision agricole.

Mots clés : Latitude, Photopériode, Modélisation, Pennisetum glaucum (L.) R. Br.), Modèle SarraH, Sénégal 
Study and integration of crop modeling to latitude effect on diverse pearl millet (Pennisetum glaucum (L.) R. Br.) genotypes' response to photoperiod in Senegal

\begin{abstract}
Objectives: The study focused on characterization of vegetative phases of different millet genotypes grown in Senegal at different latitudes and improvement of their response to photoperiod modeling. Methodology and Results: Well watered and well fertilized experiments in randomized completely blocks design with 3 replications sown at staggered dates (12 June, 10 July and 08 August) were performed in 2012 in three locations (Ndiol, Bambey and Kolda) in Senegal. Millet genotypes consisted of 2 Souna improved variety type and 6 local ecotypes which were collected in May 2011 following an increasing latitude gradient in the country. Phenology was characterized by the duration from sowing to flag leaf appearance according to planting date and latitude. Impatience phonological model had been parameterized and then used to simulate dates of panicle initiation of genotypes in the locations. The findings of the study confirmed that duration from sowing to flag leaf appearance decreased with increasing in sowing date and decreasing in latitude. Effects of latitude and sowing date on response of genotypes to photoperiod are much higher at early sowings. Simulation of vegetative phases of genotypes in Kolda and Ndiol is less accurate with parameterization of Impatience model made in Bambey. A correction equation taking in account latitude has been proposed to calculate parameters of the two location by considering their latitudes and parameters adjusted at Bambey. The procedure permitted to simulate the vegetative phases on the 2 locations with RMSE values closer to those are obtained if specific parameters were used.

Conclusions and application of findings: This study showed that most traditional genotypes are sensitive to photoperiod. The proposed correction method of Psens improved Impatience model performance. The results obtained have an operational application in crop modeling and its applications, including yield forecasting in Senegal.
\end{abstract}

Keywords: Latitude, Photoperiod, Modeling, Pennisetum glaucum (L.) R. Br), SarraH model, Senegal

\section{INTRODUCTION}

Au Sénégal, les mils locaux constituentl'essentiel de la production céréalière et occupent une place importante dans l'alimentation humaine. La région sahélienne dont fait partie le pays représente un centre important de diversité des mils. La diversification des écotypes locaux, qui s'est effectuée sur plusieurs millénaires, a permis à ces derniers de s'adapter à la variabilité spatiotemporelle des pluviométries, principale contrainte des cultures dans la région (Traoré et al., 2000). Cette variabilité entraîne en particulier une grande incertitude sur la date de semis qui, en un même site, peut se produire sur une période de près de 2 mois selon le démarrage des pluies (Sivakumar, 1988), alors que la variabilité de la fin de la saison des pluies est bien moindre (Traoré et al., 2000). De nombreux travaux ont ainsi montré que l'adaptation des écotypes traditionnels consistait en leur faculté à caler leur cycle sur les dates de début et de fin de saison des pluies, ce du fait de leur caractère photopériodique (Cochemé et Franquin, 1967; Scheuring, 1996; Reyniers, 1998). En effet, le photopériodisme permet de faire que quelle que soit la date de semis, les floraisons se déroulent plus ou moins toujours à la même date, proche de la date moyenne de la fin de la saison humide, ce qui permet de limiter les moisissures, importantes en cas de floraison trop précoce, ou les stress hydriques finaux liés aux floraisons tardives, et également de minimiser les dégâts causés par les oiseaux du fait de la synchronisation des maturités (Curtis, 1968; Vaksmann et al., 1996). Cela assure aussi un séchage efficace des panicules qui arrivent à maturité après les dernières pluies. Pourtant, suite à la péjoration de la pluviométrie de la fin des années 60 (Le Barbé et al., 2002), ce caractère a été systématiquement éliminé par les programmes de sélection pour ces zones pour créer des variétés à 
cycle fixe et plus court capables de supporter des saisons de pluies plus courtes (Dancette, 1983). Ce n'est que récemment que la photosensibilité a été réellement comprise par les sélectionneurs et agronomes comme étant un trait primordial d'adaptation à un climat fluctuant et aux contraintes biotiques de la zone sahélienne (Folliard et al., 2004; Clerget, 2004; Kouressy et al., 2008), dont les multiples avantages expliquent les préférences des paysans pour les écotypes locaux (Trouche et al., 1998). Une bonne compréhension et la modélisation du photopériodisme sont donc primordiales pour pouvoir correctement prévoir les comportements des génotypes en fonction de leur environnement et des pratiques. La modélisation de la réponse à la photopériode des mils et des sorghos tient compte des températures et durées du jour (Alagarswamy et Ritchie, 1991; Chantereau et al., 1997). Deux modèles ont fait longtemps référence: ceux de Major (1980) et de Franquin (1974). Le modèle de Major est fondé sur une augmentation linéaire de la photopériode avec la durée de la période végétative, exprimée en temps thermique, au-dessus du seuil de photopériode optimale. Le modèle de Franquin est de type hyperbolique et s'adapte bien au comportement des sorghos africains dont la durée de la phase végétative augmente de façon hyperbolique, s'accroissant rapidement au-delà d'un seuil lorsque la photopériode augmente, jusqu'à rendre impossible la floraison en jours longs. Ces modèles ne sont cependant pas adaptés lorsque le délai d'attente jusqu'à l'initiation paniculaire (IP) devient très long ou lorsque les plantes sont cultivées en dehors des périodes de semis normales (Clerget, 2004; Folliard et al., 2004). Clerget et al. (2004) ont donc proposé de tenir aussi compte du sens de variation de la durée du jour ou de ses composantes, les heures de lever et de coucher du soleil. Cette amélioration a permis une bonne simulation de la phénologie des sorghos africains dans différents environnements. Mais malgré le nombre relativement élevé de paramètres et de variables explicatives (température, photopériode, sens de variation de la durée du jour), la phénologie dans certains environnements restait inexpliquée. Pour résoudre ce problème, Folliard et al. (2004) ont proposé un modèle qui considère que les photopériodes élevées inhibent la mise à fleur et que I'IP se produit lorsque la durée du jour descend en dessous d'un seuil qui dépend de l'âge de la plante. Ces résultats ont été à la base du développement $d u$ modèle Impatience (Dingkuhn et al., 2008) qui semble à ce jour être le plus adapté aux écotypes soudano-sahéliens de mil et sorgho. Le principe du modèle Impatience est que l'initiation paniculaire se produit lorsque la photopériode descend en dessous d'un seuil qui dépend de la variété et de l'âge de la plante. Ce modèle de réponse à la photopériode $a$ été incorporé dans le modèle de simulation du développement des cultures SarraH (Dingkuhn et al., 2003). SarraH est un modèle à base écophysiologique développé par le Centre de coopération internationale en recherche agronomique pour le développement (CIRAD) en collaboration avec le Centre d'étude régional pour l'amélioration de l'adaptation à la sécheresse (Ceraas) et le Centre régional Agrhymet (CRA). II permet de simuler correctement les phénologies et les développement des biomasses et rendements des céréales, en particulier des écotypes africains traditionnels photopériodiques (Dingkuhn et al., 2008; Traoré et al., 2010). Mais récemment des études ont montré que la modélisation du phénomène n'est satisfaisante qu'à la latitude à laquelle les informations nécessaires au paramétrage du modèle ont été recueillies et qu'Impatience ne simulent pas correctement les cycles lorsque l'on change de latitude (Abdulai et al., 2012). Ce constat a mis en évidence une interaction entre la latitude et la réponse à la photopériode qui n'est pas encore comprise ni correctement intégrée à la modélisation. Par ailleurs au Sénégal SarraH n'a été, jusqu'à maintenant, paramétré que sur le Souna 3 , variété améliorée très peu photosensible, et ce sur la base d'expérimentations réalisées seulement à Bambey (Sarr et al., 2004; Siéné et al., 2010a; Siéné et al., 2010b). Notre étude a ainsi pour objectifs de caractériser les variations de cycles de différents génotypes de mil cultivés au Sénégal sous différentes latitudes et d'améliorer la modélisation de leur réponse à la photopériode en conséquence. 


\section{MATERIEL ET METHODES}

Présentation des sites de l'étude : Les essais ont été conduits lors de l'hivernage 2012 sur 3 stations expérimentales de l'Institut Sénégalais de Recherches Agricoles (ISRA), à savoir les stations de Ndiol $\left(16^{\circ} 08^{\prime} \mathrm{N}\right.$, $\left.16^{\circ} 19^{\prime} \mathrm{W}, 7 \mathrm{~m}\right)$, de Bambey $\left(14^{\circ} 42^{\prime} \mathrm{N}, 16^{\circ} 29^{\prime} \mathrm{W}, 20 \mathrm{~m}\right)$ et de Kolda $\left(12^{\circ} 51^{\prime} \mathrm{N}, 14^{\circ} 56^{\prime} \mathrm{W}, 16 \mathrm{~m}\right)$. Les sols des essais sont de types brun-rouge subaride sur sable à Ndiol, ferrugineux tropical faiblement lessivé sur sable à Bambey et ferrugineux tropical lessivé sur grès sabloargileux à Kolda. Le régime pluviométrique sur l'ensemble des sites est uni-modal avec des moyennes annuelles sur la période 1971-2000 de 189,2 mm à Ndiol, $436 \mathrm{~mm}$ à Bambey et 896,9 mm à Kolda. Les températures journalières minimales (maximales) sur la période de juin à novembre ont été en moyenne de $22,9^{\circ} \mathrm{C}\left(33,3^{\circ} \mathrm{C}\right)$ à $\mathrm{Ndiol}, 23,5^{\circ} \mathrm{C}\left(35,2^{\circ} \mathrm{C}\right)$ à Bambey et $23,2^{\circ} \mathrm{C}\left(34,7^{\circ} \mathrm{C}\right)$ à Kolda. La durée astronomique maximale du jour est de $12 \mathrm{~h} 51 \mathrm{mn} 55 \mathrm{~s}$ à Kolda, de $12 \mathrm{~h}$ $58 \mathrm{mn} 49 \mathrm{~s}$ à Bambey et de $13 \mathrm{~h} 04 \mathrm{mn} 14 \mathrm{~s}$ à Ndiol. Les écarts maximaux entre durées du jour sur la période de l'étude sont de 5'42 entre Ndiol et Bambey et 6'90 entre Bambey et Kolda (Figure 1).

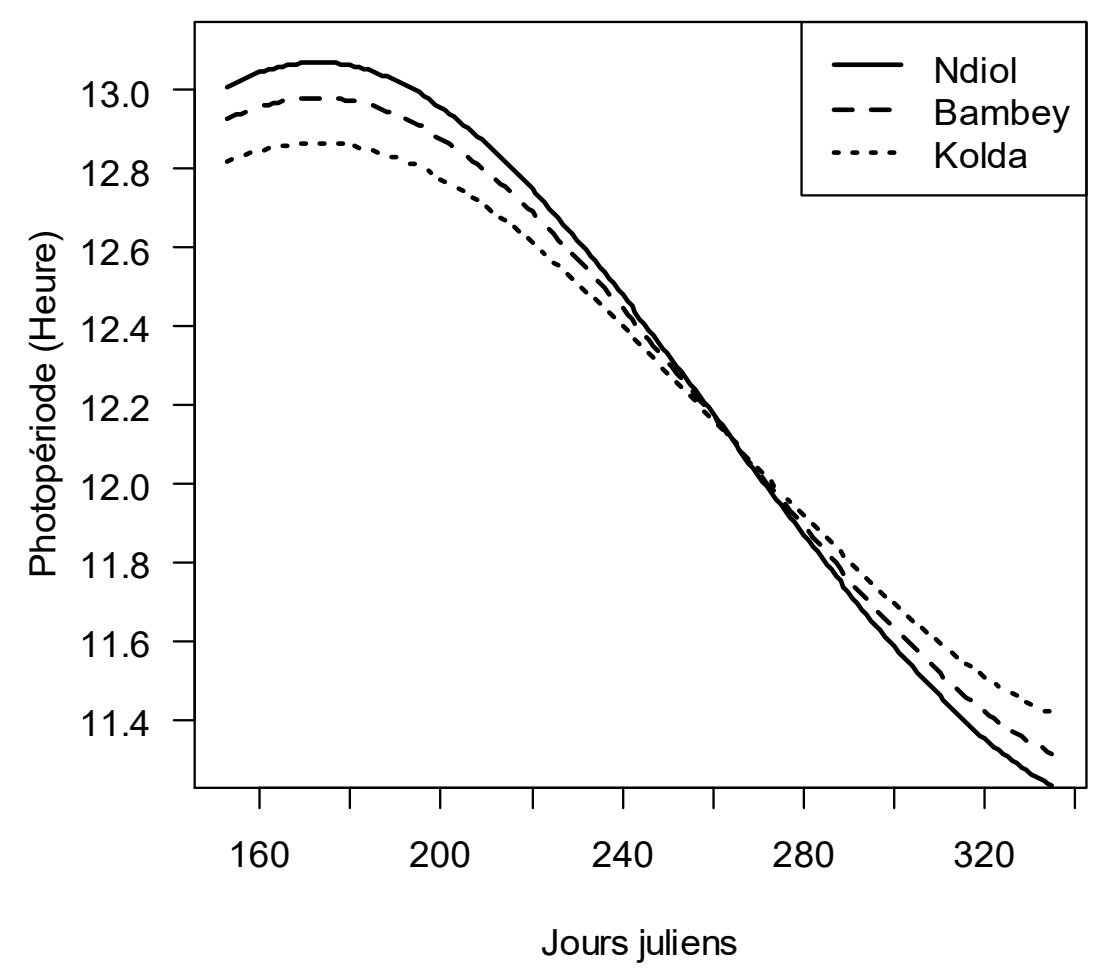

Figure 1 : Évolution de la photopériode sur les 3 sites durant la période l'étude

Matériel génétique : Le matériel végétal est composé de 2 variétés améliorées, de type Souna, et de 6 écotypes locaux dont 2 de type Souna et 4 de type Sanio, qui ont été collectés suivant un gradient latitudinal nord-sud en mai 2011 (Tableau 1). Les mils de type Souna sont des variétés précoces et peu sensibles à la photopériode. Les mils de type Sanio, dits tardifs, ont un cycle de développement long et sont très sensibles à la photopériode. Les deux variétés améliorées sont le Souna 3 (variété très vulgarisée au Sénégal) et le Thialack 2. Le Souna 3 est une population synthétique issue d'un programme de sélection. Thialack 2 est une population locale améliorée. Les 6 écotypes locaux ont été désignés par leur lieu d'origine. 
Tableau 1 : Caractéristiques des génotypes étudiés

\begin{tabular}{llll}
\hline Nom & Type & Cycle & zone d'origine ou d'adaptation \\
\hline Souna 3 & Souna amélioré & précoce & Centre-Nord \\
Thialack 2 & Thialack amélioré & intermédiaire & Centre-Sud \\
Backfassagal & Souna local & Précoce & Centre-Nord \\
Diana Bah & Souna local & Précoce & Sud \\
Séfa & Sanio amélioré & Tardif & Sud \\
Koutima & Sanio local & Tardif & Sud \\
Maka Hamdallaï & Sanio local & Tardif & Centre-Sud \\
Allahbougou & Sanio local & Tardif & Sud-Est \\
\hline
\end{tabular}

Dispositif expérimental et conduite de la culture : Sur chaque site, l'essai a été semé à 3 dates, séparées d'environ un mois, à chaque fois selon un dispositif en blocs complets randomisés à un facteur qui est le génotype et à 3 répétitions. La parcelle élémentaire est constituée de 10 poquets disposés sur 2 lignes distantes de $0,8 \mathrm{~m}$, avec un écart entre poquets de 0,8 $\mathrm{m}$. Les semis ont été effectués en humide après labour les 12 juin, 10 juillet et 08 août 2012. Des irrigations ont été effectuées afin d'éviter tout stress hydrique qui pourrait affecter les phénologies. La fertilisation a été faite selon les recommandations de l'ISRA sur chaque site. Les désherbages et luttes contre les ravageurs et maladies ont été faits à la demande. Des parcelles additionnelles de 6 lignes de 7 poquets ont été semées à Bambey pour chacun des génotypes le 13 octobre sous les mêmes conditions agronomiques en vue de faire des observations destructrices des initiations paniculaires.

Observations et paramètres calculés: Le stade phénologique observé principalement est la date d'apparition de la ligule de la feuille paniculaire, dite aussi feuille-drapeau. Les variations de longueur du cycle liées à la sensibilité à la photopériode s'expriment en effet avant son apparition, alors que la fin du cycle varie peu, ne dépendant que des températures. De plus il s'agit d'un événement facile à identifier, sans ambiguïté, ce qui n'est pas toujours le cas du stade de floraison. Les

$$
K p=\frac{S F D 1-S F D 2}{D S 2-D S 1}
$$

Avec SFD1 et SFD2, les nombres de jours entre les 2 premiers semis et DS1 et DS2, les 2 premières dates de semis. Kp varie de 0 pour les variétés insensibles à la photopériode à 1 pour les variétés strictement observations ont été faites sur chacun des 6 poquets centraux, en passant tous les deux jours dans les parcelles. Cela a permis de déterminer les durées semisligulation de la feuille drapeau (SFD). Pour déterminer la date d'initiation paniculaire (IP) dans les parcelles additionnelles, des dissections et observations visuelles ont été pratiquées régulièrement sur des pieds choisis de façon aléatoire. La période végétative sensible à la photopériode (notée ici PSP comme dans le modèle SarraH) est de durée nulle quand les plantes sont semées tardivement comme cela a été le cas pour le semis du 13 octobre. La détection de l'IP permet d'établir la durée de la phase végétative initiale non sensible à la photopériode (BVP) et celle de la phase de développement allant de l'IP à la ligulation de la feuille drapeau (IPFD). Les IPFD ont ensuite été soustraites des SFD pour avoir les durées semis-initiation paniculaire (SIP) aux différentes dates de semis. Toutes les durées calculées ont été converties en temps thermique selon l'approche broken linear function (Hammer et Muchow, 1994). Les températures de base, optimale et maximale ont été fixées respectivement à $11^{\circ} \mathrm{C}, 34^{\circ} \mathrm{C}$ et $46^{\circ} \mathrm{C}$ (Garcia-Huidobro et al., 1982; van Oosterom et al., 2001). Le coefficient de sensibilité au photopériodisme (Kp) a été calculé pour les différents génotypes selon la formule utilisée par Traoré et al. (2000).

photopériodiques pour lesquelles le raccourcissement de la période végétative compense totalement la variation entre les dates de semis (Kouressy et al., 2008). 
Analyse statistique : Le logiciel Statistique $R$ version 2.15 (R Development Core Team, 2012) a été utilisé pour les représentations graphiques et le calcul des intervalles de confiance au seuil de $5 \%$

Simulations de la réponse à la photopériode

Modèles utilisés: Le modèle de simulation du développement des cultures qui a été utilisé, SarraH

$$
\left(\frac{1000}{\sum_{i=B V P} d t t i}\right)^{\text {Pexp }} \frac{\text { Pi-Pbase }}{\text { Psat }- \text { Pbase }} \geq \text { Psens }
$$

$$
\sum_{i=B V P} d t t i
$$

représente la somme des températures de la phase végétative. Pbase et Psat sont les limites inférieure et supérieure de durée de jour au-delà desquelles l'initiation paniculaire n'est plus possible. Pi est la photopériode réelle. Pexp et Psens sont des coefficients génétiques dépendant de la variété. Le modèle est surtout sensible à la variation de Psens qui prend des valeurs proches de 0,4 pour des variétés très photopériodiques et supérieures à 1 pour des variétés insensibles à la photopériode. Pexp est généralement inférieur à 1 (Dingkuhn et al., 2008).

Paramétrage du modèle et simulations: Le paramétrage du modèle Impatience peut être effectué

\section{RESULTATS}

Effet de la date de semis sur les longueurs de cycle : La durée de la phase semis-feuille drapeau (SFD) permet bien de distinguer les Sanios, tardifs, des Sounas, précoces (Figure 2). Pour le semis du 12 juin, les génotypes tardifs ont une SFD variant de $2003^{\circ} \mathrm{C}$.j. à $1646^{\circ} \mathrm{C}$.j. à Ndiol, de $1892^{\circ} \mathrm{C}$.j. à $1561^{\circ} \mathrm{C}$.j. à Bambey et de $1768^{\circ} \mathrm{C}$.j à $1308^{\circ} \mathrm{C}$.j à Kolda. Dans ce groupe, les deux génotypes originaires du Sud du Sénégal ont eu pour l'un (Sanio de Séfa) le cycle le plus long et pour l'autre (Sanio de Koutima) le cycle le plus court. Les génotypes de type Souna ont eu des SFD variant entre $1104^{\circ} \mathrm{C}$.j. et $997^{\circ} \mathrm{C}$.j. à Ndiol, entre $1046^{\circ} \mathrm{C}$.j. à $971^{\circ} \mathrm{C}$.j. à (version 3.2) a été plusieurs fois décrit dans la littérature récente (Dingkuhn et al., 2003; Baron et al., 2005; Sultan et al., 2005; Dingkuhn et al., 2008). II intègre le modèle Impatience pour lequel l'IP est déclenchée selon les conditions suivantes :

par itération, soit en ajustant Psens et Pexp ensemble, soit en fixant Pexp à 0,17 et en ajustant uniquement Psens (Dingkuhn et al., 2008). C'est la deuxième approche que nous avons utilisée. Pour l'ensemble des variétés les seuils critique et maximal de photopériode ont été fixés respectivement à 11 heures et 13,6 heures et Pexp à 0,17. La valeur de Psens qui a permis d'obtenir la plus petite valeur de la racine carrée des erreurs quadratiques moyennes, RMSE (Kobayashi et Salam, 2000) a été retenue. Le paramétrage ainsi obtenu a été utilisé pour simuler les durées SIP sur les sites de Ndiol et de Kolda, et ceci aux 3 dates de semis.

Bambey et entre $903^{\circ} \mathrm{C}$.j. et $839^{\circ} \mathrm{C}$.j. à Kolda. Dans ce groupe, Thialack 2 a été le génotype le plus tardif. Pour l'ensemble des génotypes, la durée de la SFD est réduite au fur et à mesure que le semis est retardé. Cette réduction de cycle est beaucoup plus importante chez les génotypes tardifs. Entre les 2 premières dates de semis, elle varie de $343^{\circ} \mathrm{C}$.j. environ pour le Sanio de Séfa à $371^{\circ} \mathrm{C}$.j. pour le Sanio de Koutima. Chez les génotypes à cycle court, la réduction de cycle la plus importante est enregistrée sur Thialack $2\left(233^{\circ} \mathrm{C}\right.$.j.) $)$ et la plus faible sur le Souna de Diana Bah $\left(162^{\circ} \mathrm{C}\right.$.j.). 
Kouakou et al J. Appl. Biosci. 2013. Étude et prise compte en modélisation de l'effet de la latitude sur la réponse a la photopériode chez divers génotypes de mil du Sénégal
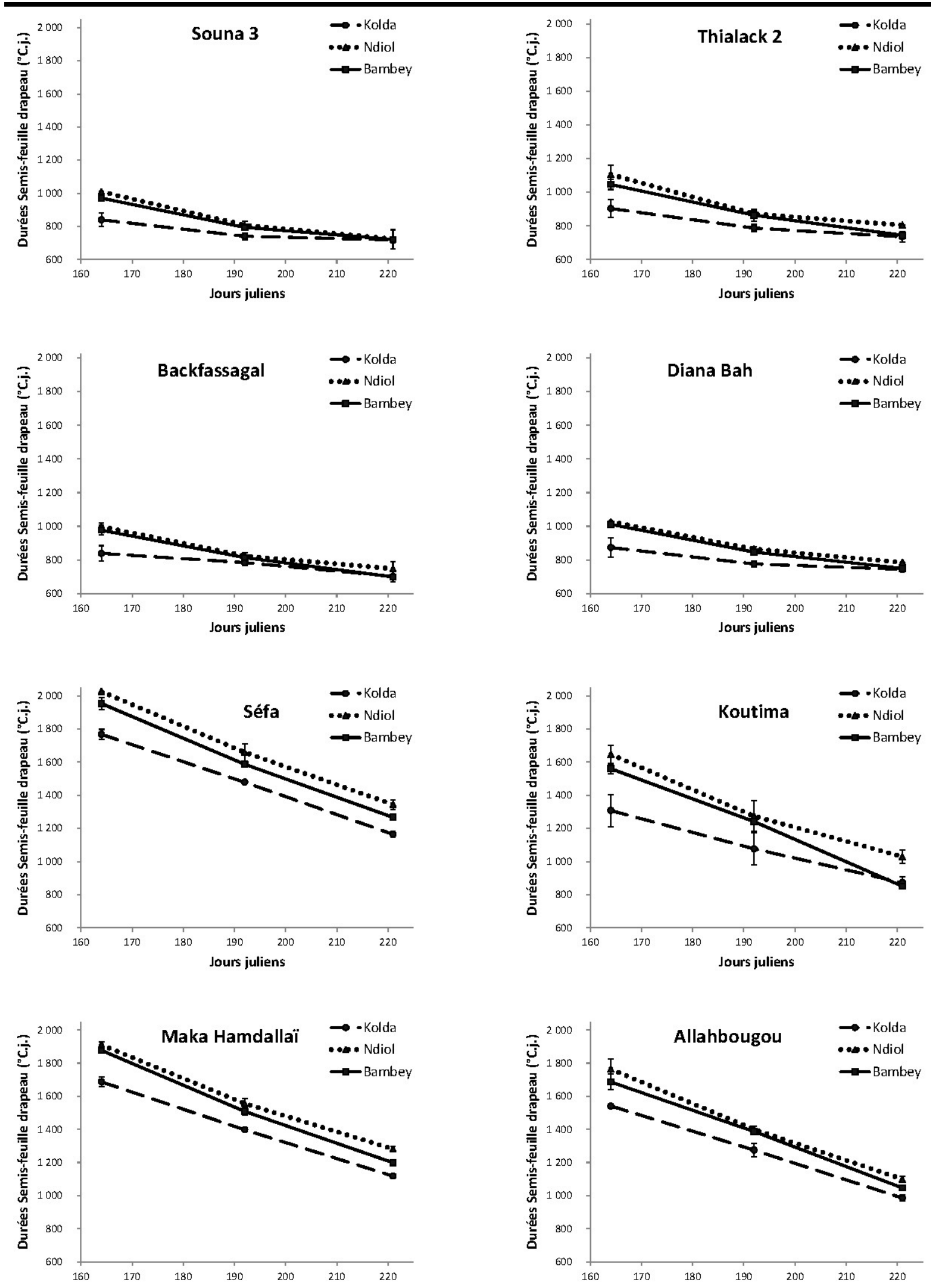

NB : Les barres d'erreurs représentent les valeurs d'intervalles de confiance calculées au seuil de $5 \%$

Figure 2 : Effet de la date de semis et de la latitude sur la durée semis-feuille drapeau $\left({ }^{\circ} \mathrm{C}\right.$.j) des 8 génotypes sur les 3 sites 
Effet de la latitude sur le photopériodisme : Les SFD de tous les génotypes varient avec la latitude (Figure 2). Les valeurs les plus élevées sont observées à Ndiol et les plus faibles à Kolda. L'effet de la latitude, plus marqué pour le semis précoce, s'estompe progressivement au fur et à mesure que le semis est retardé. Par ailleurs, la différence est plus importante entre Bambey et Kolda qu'elle ne l'est entre Bambey et Ndiol. Pour le semis du 12 juin, hormis le Sanio de Séfa où le phénomène est moins marqué, la différence sur les SFD entre Bambey et Kolda est au moins le double de celle entre Bambey et Ndiol. Pour le Souna de Diana Bah par exemple, elle est 8,5 fois plus élevée. La même tendance est observée au semis du 10 juillet où la différence sur les SFD entre
Bambey et Kolda est 2 et 10 fois supérieure à celle entre Bambey et Ndiol, respectivement pour le Sanio de Maka Hamdallaï et le Thialack 2. Toutes les variétés de l'étude ont donc montré une sensibilité à la photopériode $(K p>0)$. Les $\mathrm{Kp}$ varient en fonction de la latitude et du génotype (Figure 3). Les valeurs les plus élevées ont été observées à Ndiol et les plus faibles à Kolda. Les génotypes tardifs sont plus photopériodiques avec des Kp variant de 0,74 à 0,79 à Ndiol, de 0,58 à 0,74 à Bambey et de 0,46 à 0,60 à Kolda. Les génotypes à cycle court, faiblement photosensibles à Kolda, avec un Kp compris entre 0,08 et 0,23 , se sont avérés moyennement photosensibles à Bambey et à Ndiol avec des Kp variant entre 0,3 et 0,51 .

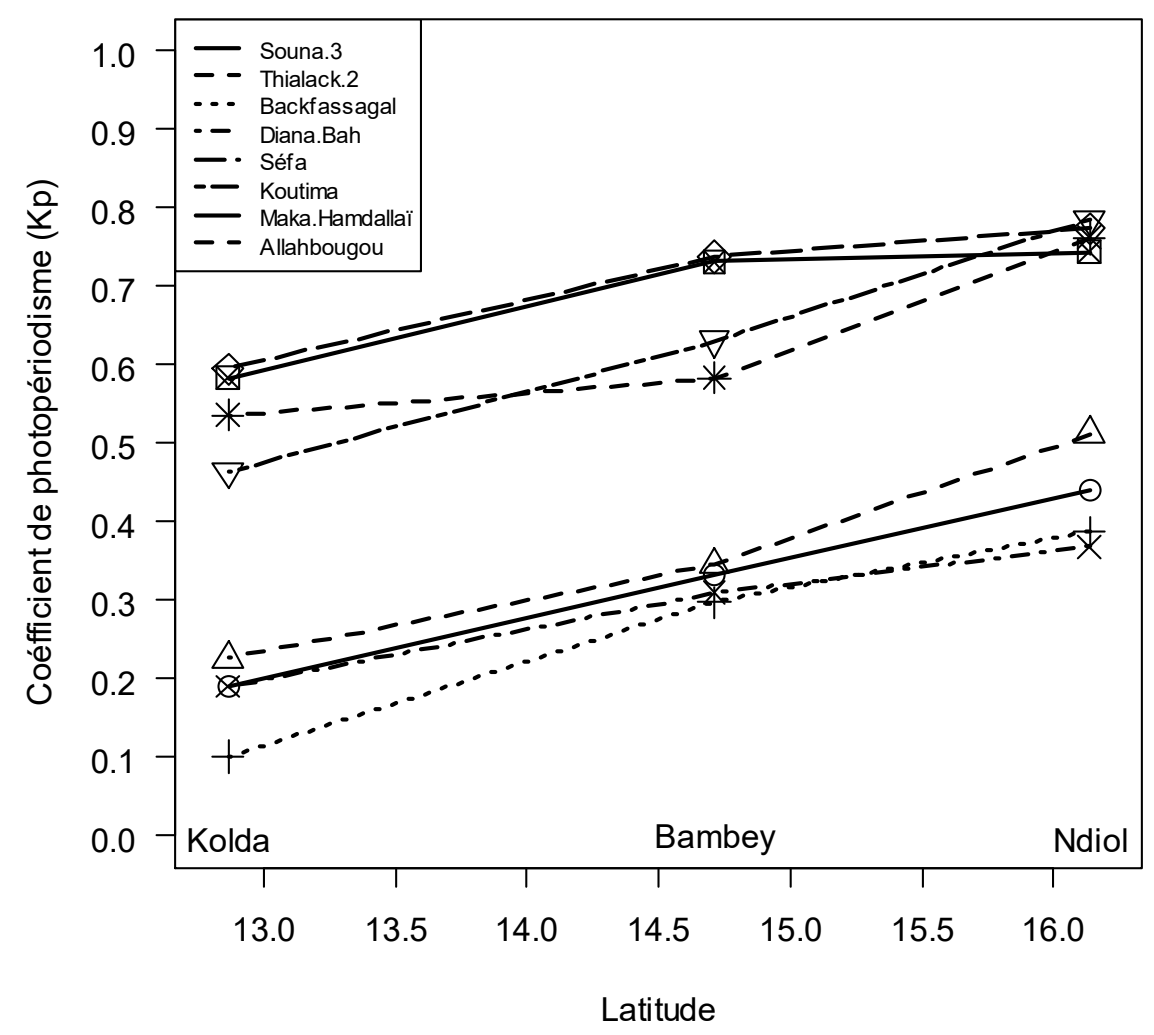

Figure 3 : Évolution du coefficient de photopériodisme des génotypes en fonction de la latitude

Amélioration de la modélisation de la réponse à la photopériode : Les durées de BVP varient de $375^{\circ} \mathrm{C}$.j. à $423^{\circ} \mathrm{C}$.j. Elles ne dépendent pas de la longueur de cycle du génotype. Les coefficients Psens diminuent quand la latitude augmente. Ils sont cependant plus élevés à Bambey qu'à Ndiol pour les variétés précoces (Tableau 2). 
Tableau 2 : Valeurs des coefficients génétiques obtenues lors du paramétrage du modèle Impatience

\begin{tabular}{lllll}
\hline Génotype & Psens (Kolda) & $\begin{array}{l}\text { Psens } \\
\text { (Bambey) }\end{array}$ & Psens (Ndiol) & $\begin{array}{l}\text { Phase végétative de base } \\
\left({ }^{\circ} \text { C.j.) }\right.\end{array}$ \\
\hline Souna 3 & 0,80 & 0,76 & 0,79 & 386 \\
Thialack 2 & 0,85 & 0,79 & 0,81 & 386 \\
Backfassagal & 0,80 & 0,77 & 0,81 & 403 \\
Diana Bah & 0,82 & 0,76 & 0,81 & 422 \\
Séfa & 0,45 & 0,41 & 0,40 & 423 \\
Koutima & 0,55 & 0,50 & 0,48 & 375 \\
Maka Hamdallaï & 0,45 & 0,41 & 0,41 & 408 \\
Allahbougou & 0,46 & 0,43 & 0,43 & 375 \\
\hline
\end{tabular}

L'ajustement des paramètres effectué sur les résultats obtenus sur chaque site permet de simuler correctement les durées SIP des variétés du même site aux 3 dates de semis, avec des valeurs de RMSE de 2,73 jours, 1,96 jours et 2,24 jours respectivement à Ndiol, Bambey et Kolda (Figure 4).

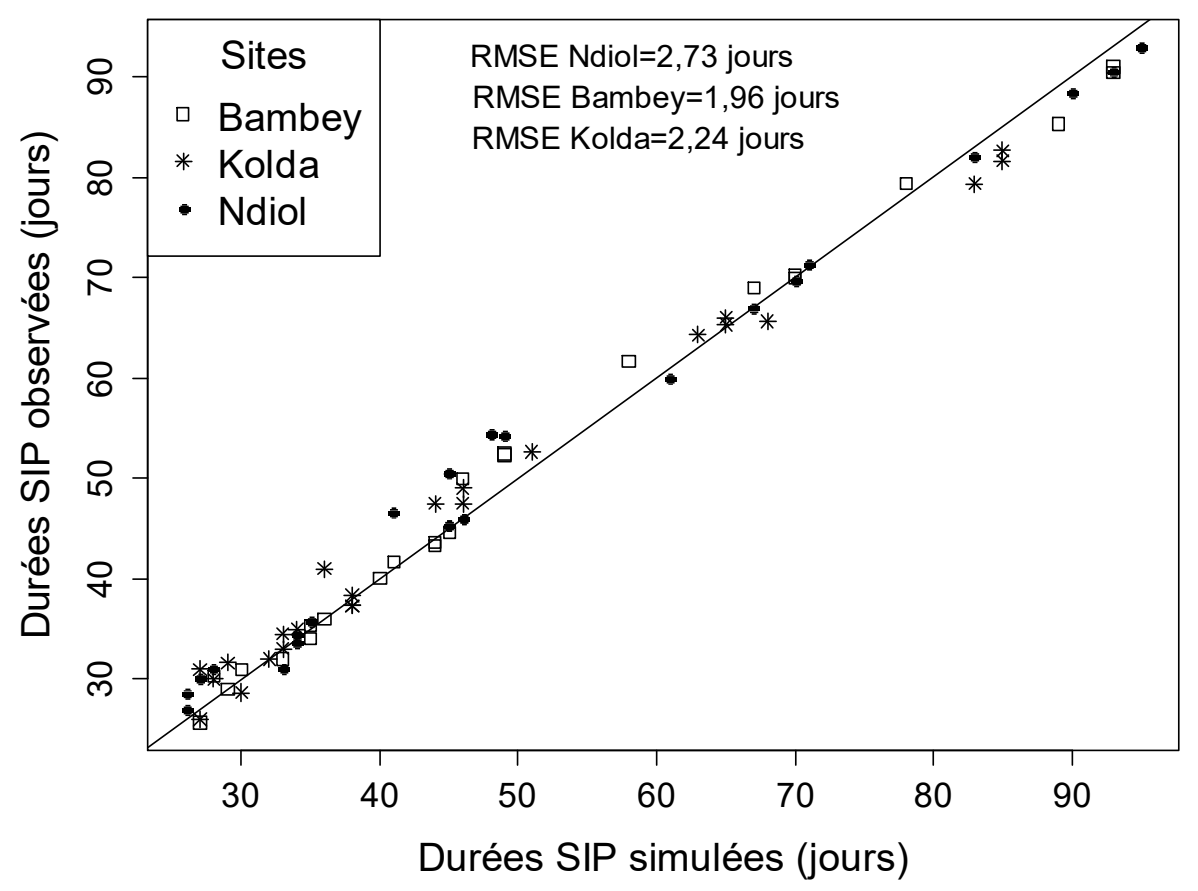

Figure 4 : Durées semis-initiation paniculaire (SIP) simulées avec le paramétrage obtenu sur chaque site en fonction des durées semis-initiation paniculaire (SIP) observées correspondantes

Lorsque le paramétrage obtenu sur l'un des sites est utilisé pour effectuer les simulations sur les 2 autres, les résultats sont moins bons : par exemple si on utilise le paramétrage établi à Bambey, on obtient des RMSE de 4,18 jours pour Ndiol et 5,19 jours à Kolda. La méthode de correction proposée pour le sorgho au Mali (Abdulai et al., 2012) a donc été utilisée. Elle consiste à établir, à partir des différentes valeurs de Psens déterminées sur les différents sites et des latitudes des sites, une équation de régression permettant de déterminer la valeur de
Psens d'un site en fonction de sa latitude et d'une valeur de référence de Psens établie dans un autre site. La méthode de correction a été développée en considérant chacun des 3 sites et en testant sur les 2 autres. Les meilleurs résultats sont obtenus quand on considère Bambey comme site de référence, ce qui s'explique par le fait qu'il soit central, mais quel que soit le site de référence, la méthode de correction permet d'améliorer les ajustements. L'équation établie pour estimer le Psens 
d'une variété en un site quelconque à partir de sa latitude et de son Psens déterminé à Bambey est la suivante :

PsensSite

$=1,2468 \times$ PsensBambey $-0,0120 \times$ PsensBambey $\mathrm{x}$ LatSite $-0,0148 ; R 2=0,99$

avec PsensSite = coefficient génétique de photosensibilité pour le site, PsensBambey = coefficient génétique de photosensibilité à Bambey et LatSite = latitude du Site.

Les paramétrages estimés ainsi ont été utilisés pour simuler les SIP sur les sites de Ndiol et Kolda. Cela améliore les simulations des durées SIP avec des valeurs de RMSE proches de celles obtenues lorsque l'on utilise les paramétrages spécifiques de chaque site (Figure 5).
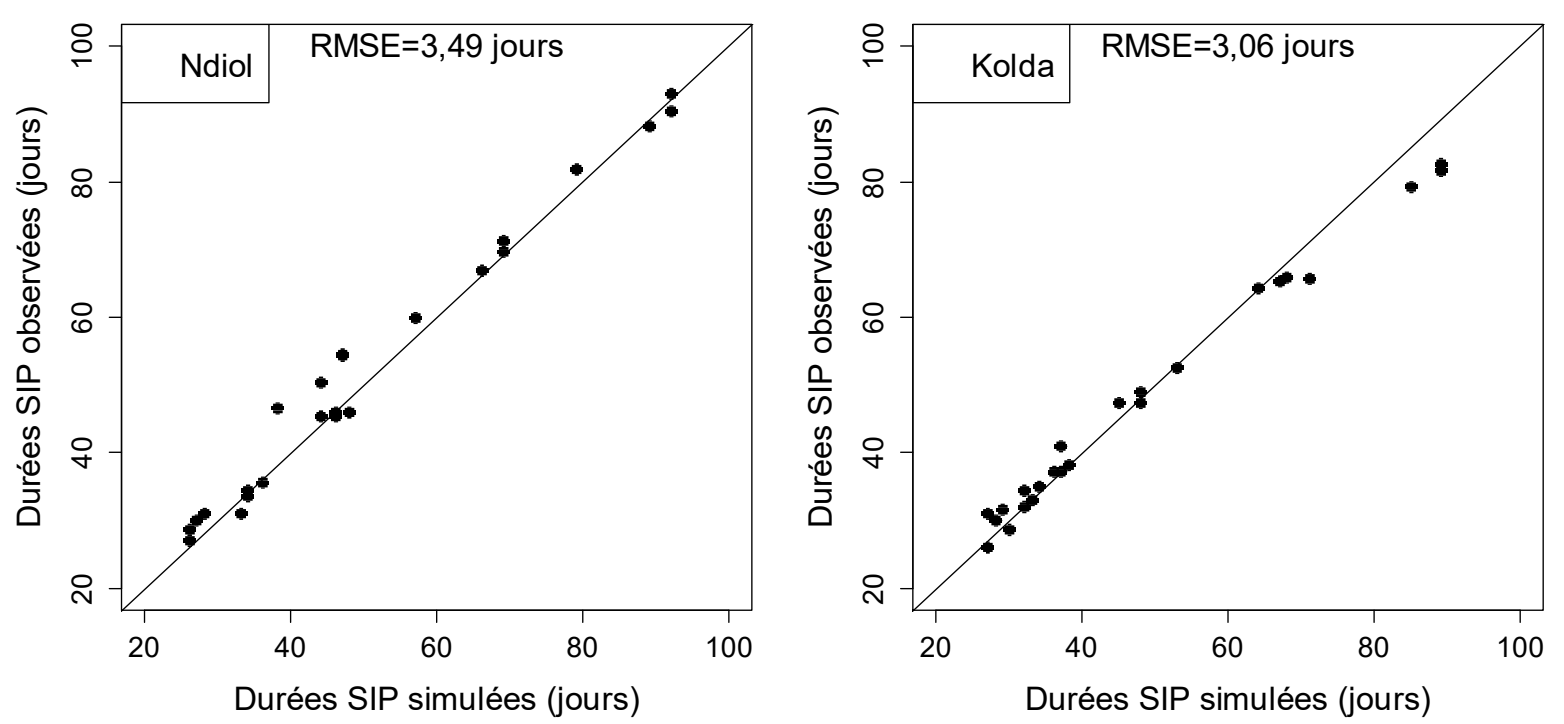

Figure 5 : Durées semis-initiation paniculaire (SIP) simulées avec le paramétrage ajusté à Ndiol et à Kolda en fonction des durées semis-initiation paniculaire (SIP) observées correspondantes

\section{DISCUSSION}

L'étude a porté sur la caractérisation des variations de cycles de génotypes de mil en fonction des dates de semis et de la latitude en vue d'améliorer la modélisation de leur phénologie. L'originalité de la méthodologie réside dans l'utilisation d'un modèle de développement de culture en complément d'expérimentations multi-locales et à plusieurs dates de semis pour l'ajustement des paramètres du modèle Impatience. Le dispositif est similaire à ceux utilisés au Mali par les équipes de I'Institut d'Économie Rurale (IER) et du CIRAD qui travaillent depuis des années sur le photopériodisme (Vaksmann et al., 1996; Kouressy et al., 2008; Abdulai et al., 2012). Au Sénégal, c'est la toute première expérience d'étude de la sensibilité à la photopériode et d'ajustement du coefficient Psens d'un lieu à partir de sa latitude et du Psens d'un site de référence. Contrairement aux travaux de (Curtis, 1968) au Nigéria, qui a montré que la date d'initiation paniculaire des variétés traditionnelles était indépendante de la latitude, les 8 variétés de l'étude (précoces et tardives) ont vu leurs durées SIP se raccourcir sous les faibles latitudes. Ce résultat, similaire à ceux de Vaksmann et al. (1996), Kouressy et al.(2008) et Abdulai et al.(2012)), s'explique par le fait que ces variétés étant de jours courts, et la durée du jour étant plus longue au nord sur la période de l'étude, cela entraîne une diminution de la durée de la période végétative lorsque l'on déplace une expérimentation du nord au sud. Ainsi, comme l'ont montré Kouressy et al (2008), les variétés semées à basse latitude se montrent plus précoces, leur floraison est accélérée et leur cycle écourté. La durée SIP a également diminué au fur et à mesure que le semis était retardé. Ce phénomène est dû au fait que pour les semis précoces la durée du jour est trop élevée pour permettre l'initiation de la panicule ; alors la période végétative se poursuit jusqu'à ce que la durée du jour soit devenue suffisamment courte. Ce résultat est conforme à ceux obtenus par Folliard et al. (2004) et Kouressy et al. (2008). Par ailleurs, une étude menée par 
Vaksmann et al. (1998) a montré que les variétés originaires du Nord (haute latitude) étaient plus photopériodiques que celles du Sud, ce qui n'est pas le cas des variétés de notre étude dont les plus photopériodiques étaient en grande partie celles provenant du Sud. Mais nous ne pouvons rien en conclure car nous avons travaillé sur des échantillons trop restreints. Un plus grand nombre de variétés provenant de différentes latitudes aurait pu permettre de dégager une tendance claire sur la réaction photopériodique des génotypes en fonction de leur aire d'origine. L'ajustement du coefficient Psens est très

\section{CONCLUSION}

L'étude a permis de montrer que la plupart des variétés traditionnelles, qu'elles soient précoces ou tardives, sont plus ou moins photosensibles. Leur longueur de cycle augmente avec la latitude et diminue avec la date de semis. Le coefficient génétique (Psens) d'une variété en un lieu donné peut être déterminé en fonction de la latitude de ce lieu et du Psens d'un lieu de référence. $\mathrm{Ce}$

\section{REMERCIEMENTS}

Cette étude a été réalisée grâce à l'appui financier du Programme de Productivité Agricole en Afrique de l'Ouest (PPAAONAAPP). Nos remerciements vont à l'endroit du Deutscher Akademischer Austausch Dienst (DAAD) pour

\section{REFERENCES}

Abdulai AL, Kouressy M, Vaksmann M, Asch F, Giese M et Holger B. 2012. Latitude and date of sowing influences phenology of photoperiod-sensitive sorghums. Journal of Agronomy and Crop Science 198 (5): 340-348

Alagarswamy $G$ et Ritchie JT. 1991. Phasic development in CERES-Sorghum model. In T Hodges (ed.). CRC Press. Boca Raton, Florida (USA). p 143-152

Le Barbé L., Lebel T et Tapsoba D. 2002. Rainfall Variability in West Africa during the Years 1950 90. Journal of Climate 15 (2): 187-202

Baron C, Sultan B, Balme M, Sarr B, Traore S, Lebel T, Janicot S et Dingkuhn M. 2005. From GCM grid cell to agricultural plot: scale issues affecting modelling of climate impact. Philosophical Transactions of the Royal Society B: Biological Sciences 360 (1463): 2095-2108

Chantereau J, Vaksmann M, Bahmani I, Hamada M, Chartier M et Bonhomme R. 1997. Characterization of different temperature and photoperiod responses in African sorghum simple et trouve une application opérationnelle en modélisation des cultures et ses applications. Elle donne un paramétrage acceptable permettant ainsi de s'affranchir de la reprise de la détermination de Psens à chaque latitude à travers des expérimentations coûteuses en temps et en argent. Le rôle de la latitude est très déterminant dans la procédure d'ajustement mais reste encore incompris comme le soulignent Abdulai et al. (2012). Des études sont donc nécessaires afin de déterminer les mécanismes physiologiques qui soustendent son action.

résultat trouve une application opérationnelle en modélisation des cultures et ses applications dont la prévision agricole en ce sens que la simulation des rendements peut être effectuée sous différentes latitudes, sans être obligé d'effectuer de nouvelles expérimentations pour le paramétrage de la phénologie.

la bourse octroyée au premier auteur. Nos remerciements vont également à l'endroit des techniciens du CRA de Ndiol, du CNRA de Bambey et du CRZ de Kolda pour leur appui dans la collecte des données

cultivars. In Ratnadass A, Chanterau J, Gigou J (eds.). CIRAD, Montpellier (France), Bamako (Mali). p 29-35

Clerget B, Dingkuhn M, Chantereau J, Hemberger J, Louarn $\mathrm{G}$ et Vaksmann M. 2004. Does panicle initiation in tropical sorghum depend on day-today change in photoperiod? Field Crops Research 88 (1): 21-37

Clerget B. 2004. Le rôle du photopériodisme dans l'élaboration du rendement de trois variétés de sorgho cultivées en Afrique de l'Ouest. Thèse de doctorat, Institut National Agronomique ParisGrignon, Paris-Grignon (France). 192 pp

Cochemé J et Franquin P. 1967. An agroclimatology survey of a semiarid area in Africa South of the Sahara. Secretariat of the World Meteorological Organization. 174 pp

Curtis DL. 1968. The relation between the date of heading of Nigerian sorghums and the duration of the growing season. Journal of Applied Ecology 5 (1): 215-226

Dancette C. 1983. Besoins en eau du mil au Sénégal. 
Adaptation en zone semi-aride tropicale. Agronomie Tropicale 38 (4): 267-280

Diarra B et Konaré K. 1994. Réduction de l'impact du climat sur le calendrier agricole au Sahel. In Bilan hydrique agricole et sécheresse en Afrique tropicale. Reyniers FN, Netoyo L (eds.). John Libbey, Paris (France). p 31-49

Dingkuhn M, Baron C, Bonnal V, Maraux F, Sarr B, Sultan B, Clopes A et Forest F. 2003. Decision support tools for rainfed crops in the Sahel at the plot and regional scales. In Decision Support Tools for Smallholder Agriculture in SubSaharan Africa-A practical Guide. Struif Bontkes TE et Wopereis MCS. CTA, IFDC Wageningen (The Netherlands). p 127-139

Dingkuhn M, Kouressy M, Vaksmann M, Clerget B et Chantereau J. 2008. A model of sorghum photoperiodism using the concept of thresholdlowering during prolonged appetence. European Journal of Agronomy 28 (2): 74-89

Folliard A, Traoré PCS, Vaksmann $M$ et Kouressy $M$. 2004. Modeling of sorghum response to photoperiod: a threshold-hyperbolic approach. Field Crops Research 89 (1) : 59-70

Franquin P. 1974. Formulation des phénomènes apparents de photothermopériodisme en conditions naturelles. Principes de base. Cahiers ORSTOM, série Biologie. 23: 31-43

Garcia-Huidobro J, Monteith JL et Squire GR. 1982. Time, Temperature and Germination of Pearl Millet (Pennisetum Typhoides S. \& H.) I. Constant temperature. Journal of Experimental Botany 33 (2): 288-296

Hammer GL et Muchow RC. 1994. Assessing climatic risk to sorghum production in water-limited subtropical environments I. Development and testing of a simulation model. Field Crops Research 36 (3): 221-234

Kazuhiko K et Salam MU. 2000. Comparing simulated and measured values using Mean Squared Deviation and its components. Agronomy Journal 92 (2): 345-352

Kouressy M, Traoré SB, Vaksmann M, Grum M, Maikano I, Soumare M, Traoré PS, Bazile D, Dingkuhn M et Sidibé A. 2008. Adaptation des sorghos du Mali a la variabilité climatique. Cahiers Agricultures 17 (2): 95-100

Kouressy M, Dingkuhn M, Vaksmann $M$ et Heinemann AB. 2008. Adaptation to diverse semi-arid environments of sorghum genotypes having different plant type and sensitivity to photoperiod. Agricultural and Forest Meteorology 148 (3): 357-371

Major DJ. 1980. Photoperiod response characteristics controlling flowering of nine crop species. Canadian Journal of Plant Science 60 (3): 777784

Van Oosterom EJ, Carberry PS, Hargreaves JNG et O'Leary GJ. 2001. Simulating growth, development, and yield of tillering pearl millet: II. Simulation of canopy development. Field Crops Research 72 (1) : 67-91

R Development Core Team. 2012. R: A language and environment for statistical computing. $R$ Foundation for Statistical Computing. Windows (version 2.15.0). CRAN, Vienna (Austria)

Reyniers FN. 1998. Présentation du projet DHMC et des objectifs du séminaire final: pour une approche écosystémique de l'amélioration des écotypes locaux de sorgho et de mil. In Actes du séminaire final, 27-30 avril 1998, Florence, Bacci L, Reyniers FN (eds.). Cesia, Florence (Italie), CIRAD, Montpellier (France): p 21-28

Reyniers FN, Waneukeum V, Vaksmann M et Kouressy M. 1998. Effet de la latitude sur le ratio grain/paille des écotypes de mil au Mali: conséquences pour la sélection. In Actes du séminaire final, 27-30 avril 1998, Florence, Bacci L, Reyniers FN (eds.). Cesia, Florence (Italie), CIRAD, Montpellier (France): p 79-93

Sarr B, Baron C, Diouf O, Dingkuhn M, Braconnier S, Dieng I, Fall M et Sylla CY. 2004. Modélisation de la croissance et de la productivité des cultures céréalières, mise au point d'un SIG pour l'amélioration des systèmes de prévisions agricoles au Sénégal. Rapport technique final 17. Ceraas-CIRAD, Thiès (Sénégal)

Scheuring JF. 1996. The dilemma of daylength. In Lost Crops of Africa, National Academy Press, Volume I: Grains. National Research Council. Washington (USA). $54 \mathrm{pp}$

Siéné LAC, Muller B et Aké S. 2010a. Effet de la densité de semis sur l'évolution du coefficient d'extinction de la lumière et de l'efficience de conversion du rayonnement absorbé par Le couvert végétal de deux variétés de mil de longueur de cycle différente. International Journal of Biological and Chemical Sciences 4 (5):1462-1479

Siéné LAC, Muller B et Aké S. 2010b. Étude du développement et de la répartition de la biomasse chez deux variétés de mil de longueur 
de cycle différente sous trois densités de semis. Journal of Applied Biosciences 35: 2260-2278.

Sivakumar MVK. 1988. Predicting rainy season potential from the onset of rains in southern sahelian and soudanian climatic zones of West Africa. Agricultural and Forest Meteorology 42 (4): 295305

Sultan B, Baron C, Dingkuhn M, Sarr B et Janicot S. 2005. Agricultural impacts of large-scale variability of the West African monsoon. Agricultural and Forest Meteorology $128(1-2)$ : 93-110

Traoré SB, Reyniers F, Vaksmann M, Koné B, Sidibe A, Yorote A, Yattara K et Kouressy M. 2000. Adaptation a la sécheresse des écotypes locaux de sorghos du Mali. Sécheresse 11 (4): 227-237

Traoré SB, Alhassane A, Muller M, Kouressy M, Somé L, Sultan B, Oettli $P$, Siéné LAC, Sangaré $S$, Vaksmann M, Diop M, Dingkhun M, Baron C. 2010. Characterizing and modeling the diversity of cropping situations under climatic constraints in West Africa. Atmospheric Science Letters 12 (1): 89-95

Trouche G, Chantereau J et Zongo JD. 1998. Variétés traditionnelles et variétés améliorées de sorgho dans les régions sahéliennes. In Actes du séminaire final, 27-30 avril 1998, Florence, Bacci L, Reyniers FN (eds.). Cesia, Florence (Italie), CIRAD, Montpellier (France)

Vaksmann M, Traoré SB et Niangado O. 1996. Le photopériodisme des sorghos africains. Agriculture et Développement 9: 13-18

Vaksmann M, Traoré SB, Kouressy M, Coulibaly $\mathrm{H}$ et Reyniers FN. 1998. Étude du développement d'un sorgho photopériodique du Mali. In Actes du séminaire final, 27-30 avril 1998, Florence, Bacci L, Reyniers FN (eds.). Cesia, Florence (Italie), CIRAD, Montpellier (France): p 109-122 